DOI: http://doi.org/10.21698/simi.2018.ab10

\title{
NEW PROMISING HYBRID MATERIAL BASED ON MONTMORILLONITE FOR METAL IONS REMOVAL FROM CONTAMINATED WATER
}

\author{
$\underline{\text { Iolanda-Veronica Ganea }}{ }^{1,2}$, Alexandrina $\mathrm{Nan}^{2}$, Carmen Roba $^{1}$, Rodica Turcu ${ }^{2}$ \\ ${ }^{1}$ Faculty of Environmental Science and Engineering, "Babeș-Bolyai" University, 30 Fântânele, \\ 400294, Cluj-Napoca, ganea.yolanda@yahoo.com, robaacarmen@yahoo.com, Romania \\ ${ }^{2}$ National Institute for Research and Development of Isotopic and Molecular Technologies, 67- \\ 103 Donat, 400293, Cluj-Napoca, alexandrina.nan@itim-cj.ro, rodica.turcu@itim-cj.ro, Romania
}

Keywords: montmorillonite, heavy metal contamination, poly(benzofurane-coarylacetic acid), water treatment

\section{Introduction}

In the last four decades, environmental protection forums stated that economical systems could be considered sustainable only if the resources deterioration does not exceed environment's capacity to regenerate. Heavy metal pollution due to rapid industrialization and growth in world population has become a serious threat to environment and all forms of life. The persistent nature of these compounds raises even more significant toxicological concerns for ecosystems, and thereby human health. Thus, researchers have been focusing on developing new materials through "green chemistry methods" in order to remove this type of pollutants from the environment. Unfortunately, conventional methods of removing metal ions from water have become inefficient in the context of mandatory compliance with decreasing the concentrations under European standards and national water quality limit values. Therefore, synthesizing new materials based on polymers with chelating and absorption properties represents a viable and useful alternative to classic decontamination methods, considering the cost-benefit ratios and the high absorption capacity of retaining toxic metal ions. We report here the modification of montmorillonite (MMT) with poly (benzofurane-co-arylacetic) acid (PBAAA) functionalized with dopamine (DA) able to complex metal ions.

\section{Materials and methods}

MMT was suspended in a beaker containing a mixture of methanol and deionized water; furthermore PBAAA was dissolved in the formed suspension (Scheme 1). The suspension was ultrasonicated for $1 \mathrm{~h}$ and afterwards was kept into an oven at $160{ }^{\circ} \mathrm{C}$ for $6 \mathrm{~h}$, resulting a bright lilac solid, namely MMT-PBAAA which was further purified. In order to increase the chelating potential of the MMT-PBAAA, we attached DA at the polymer chain. Therefore, MMT-PBAAA and DA were placed in a flask, the suspension was refluxed for 2 days (Scheme 1) and the dark grey precipitate MMT-PBAAA-DA was filtered and purified, analyzed and used for water depollution. 


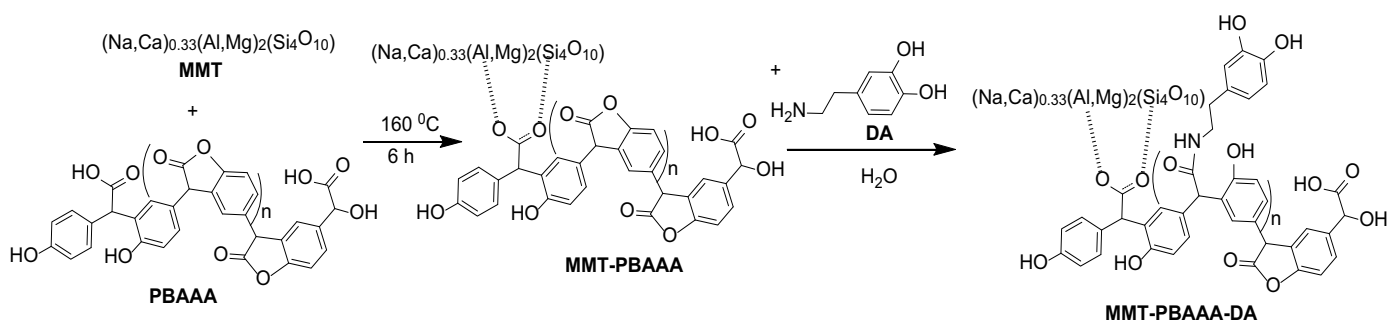

Scheme 1. Preparation of MMT-PBAAA-DA

The synthesized material was structural investigated by SEM, EDX, TGA, XPS, FTIR and the heavy metals were determined by AAS analysis. The resulting hybrid material was further applied for $\mathrm{Cu}^{2+}, \mathrm{Zn}^{2+}, \mathrm{Mn}^{2+}, \mathrm{Fe}^{3+}, \mathrm{Pb}^{2+}, \mathrm{Cd}^{2+}, \mathrm{Cr}^{3+}, \mathrm{Ni}^{2+}$ absorption from stock solutions of $40 \mathrm{mg} / \mathrm{L}$ and $100 \mathrm{mg} / \mathrm{L}$ concentrations and also from contaminated water samples collected from Roșia Montană Mining Area.

\section{Results and conclusions}

The FTIR spectrum and the TGA diagram of MMT-PBAAA-DA prove the attachment of the polymer on the MMT surface. Significant changes can be noticed regarding the physico-chemical parameters of the solutions after absorption: electrical conductivity increases while the $\mathrm{pH}$ decreases. The absorption percentages were excellent for all the metal ions investigated. The synthesized material shows selectivity for example for $\mathrm{Pb}^{2+}, \mathrm{Cd}^{2+}$ (Figure 1).

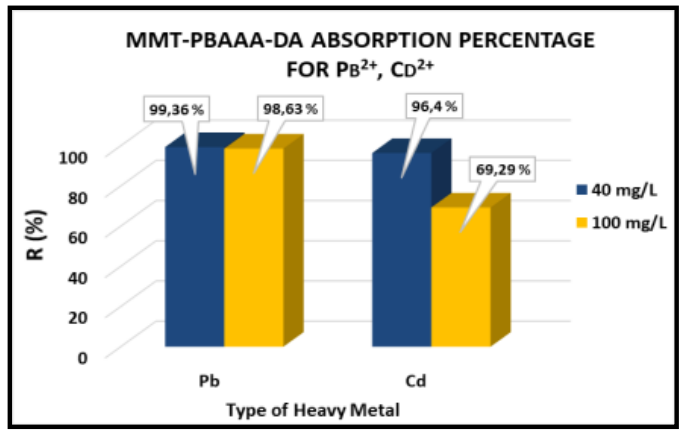

Figure 1. Absorption percentages obtained in the case of $\mathrm{Pb}^{2+}$ and $\mathrm{Cd}^{2+}$

In conclusion, a new type of hybrid material based on MMT and PBAAA was developed through an extremely easy and non-catalytic synthesis. The resulting material can be prepared with low costs, easily separated by filtration and exhibits good absorption properties which makes it attractive for applications in water treatment.

\section{Acknowledgements}

This work was supported by Romanian Nucleu Programme, Project PN 180302 03. We wish to acknowledge Dr. Monica Dan and PhD Porav Sebastian from National Institute for Research and Development of Isotopic and Molecular Technologies Cluj-Napoca for conducting TGA measurements and SEM images. We also thank to Prof. Dr. Eugen Gurzău and Chem. Angela Vâlcan from Environmental Health Center for AAS measurements. 